\title{
Necrotizing Enterocolitis
}

National Cancer Institute

\section{Source}

National Cancer Institute. Necrotizing Enterocolitis. NCI Thesaurus. Code C84915.

A fulminating disease of neonates in which there is extensive mucosal ulceration, pseudomembrane formation, submucosal hemorrhage, and necrosis usually of the right colon, cecum, terminal ileum, and appendix, possibly due to perinatal intestinal ischemia and bacterial invasion. Progression can lead to necrosis, perforation and/or scarring of the intestinal tract. 